\title{
A Study on Deep Learning and Its Enlightenment on China's Foreign Language Learning
}

\author{
Wang Honglin \\ Sichuan University/ Zhejiang Wanli University
}

\begin{abstract}
In recent years, deep learning has drawn extensive attention from experts both at home and abroad, involving multidisciplinary fields such as learning science, foreign language learning and so on. The study starts from the theoretical framework of deep learning, and then focuses on relevant foreign studies, such as the factors affecting deep learning, application of deep leaning in multidisciplinary fields, such as learning science, and foreign language learning. Based on the above analysis, the study emphasizes on the enlightenment of deep learning on China's foreign language learning and predicts further study directions in the future.
\end{abstract}

Keywords-Deep learning; Multidisciplinary studies; Foreign language learning in China; Enlightenment

\section{INTRODUCTION}

Deep learning stems from the research on artificial neural network and has been widely used in computer science, machine learning, artificial intelligence, biotechnology, cognitive neuroscience, natural language processing, image processing, face recognition, and speech recognition in recent years (Hinton 2012; Postareff 2015; Najafabad 2016). The study of deep learning in the field of artificial intelligence has been very hot in recent years, for example, Baidu established an in-depth research center, Google hired Geoffrey Hinton, the father of "deep learning", adopts deep learning in exploring artificial intelligence (Duan Jinju, Yu Shengquan 2013). Some companies in China apply deep learning in speech recognition, speech synthesis, and speech evaluation, thus developing some foreign language learning and interpreting application software. Meanwhile, deep learning has also attracted attention in interdisciplinary areas such as learning science and foreign language learning.

The concept of deep learning relative to surface learning was first proposed by foreign scholars (Marton \& Saljc 1976). In contrast, surface learning focuses on low-order thinking capabilities such as memory, understanding, and applications. Deep learning focuses on higher-order thinking capabilities such as analysis, evaluation, and innovation (Duan Jinju, Yu Shengquan 2013). Some scholars have suggested that deep learning has many advantages compared with surface learning (Liu Jianwei et al. 2014). In summary, deep learning pays more attention to the cultivation of higher-order thinking ability and problem-solving ability, and more in line with the learning characteristics of the information age.

Then, what is the deep learning adopted in foreign language learning in China? What is its feasibility and appropriateness?
These are issues concerning this study. Before these questions are answered, it is necessary to reflect on the current situation and existing problems of foreign language learning in China. In recent years, with the implementation of national strategies such as "The Belt and Road Initiative", foreign language learning has received unprecedented attention, and has made great strides in the number and size of learners. However, there are still many problems existing in current English learning, such as severe academic separation, poor language output, poor literacy, and weak thinking ability (Wen Qiufang 2017), among which, the most prominent is the problem of separation of learning and application.

Although the academic community is aware of the existing problems in the foreign language learning, and have attempted to find the root cause of the problem and to propose solutions, no best solution has been yet found. To solve the problem appropriately, it is necessary to integrate theoretical research and practical exploration. In this kind of situation, deep learning would be a good perspective to solve the existing problems in foreign language education. Unfortunately, there is little research on foreign language learning by applying deep learning theory. In this context, this study focuses on the latest development of deep learning, and concentrates on its implication on foreign language learning in China so as to predict its future research trends, thus providing a new perspective for the study of foreign language learning in China.

\section{THEORETICAL FRAMEWORK OF DEEP LEARNING}

Deep learning has received extensive attention in the interdisciplinary field. The following sections will examine the concepts of deep learning, its influencing factors, and relevant research in interdisciplinary areas such as learning science and foreign language learning.

\section{A. Definition of Deep Learning}

It is generally believed that deep learning focuses on critical thinking, problem solving, cooperation and self-directed learning, the association of old and new knowledge, and the relationship between learners and the real world. The concept of deep learning was first proposed by Marto, F. and Saljc, R. (1976) as opposed to surface learning. Some scholars believe that deep learning is a learning style that focuses on critical analysis, the connection between concepts, creative problemsolving, and application of knowledge (Harvey \& Kamvounias 2008). Some studies define deep learning from a constructivist perspective as the following: "Deep learning is learning based 
on understanding. It emphasizes that learners critically learn new ideas and new knowledge, and incorporate them into their original cognitive structures. The original knowledge is transferred to a new situation so as to help make decisions and solve problems (Jiao Jianli, Jia Yimin 2011:39). In short, deep learning refers to meaningful and active learning. Some scholars have also suggested that deep learning focuses on critical understanding, emphasizing information integration, promoting knowledge construction, emphasizing migration applications, problem solving, and proactive lifelong learning.

However, some researchers consider application competence as higher-order thinking, and the solution to the problem and the application of knowledge are the core of deep learning (Harvey \& Kamvounias 2008). There are also studies that classify applications into low-order thinking skills, propose surface learning to focus on low-order thinking such as memory, understanding, and application, while deep learning focuses on higher-order thinking such as analysis, evaluation, and innovation (Duan Jinju, Yu Shengquan 2013). The author believes that whether the application ability is categorized as surface learning or deep learning, it can be affirmed that application ability plays a crucial role in the process of transition from surface learning to deep learning. It is to connect surface learning and deep learning. To sum up, deep learning is an active and active learning style, emphasizing the construction and connectivity of knowledge, focusing on the solution of practical problems, as well as highlighting the application of knowledge.

\section{B. Factors Affecting Deep Learning}

Since the concept of deep learning was proposed, scholars have carried out related research from different perspectives. The most prominent one is the research on the influencing factors of deep learning, which mainly focuses on internal and external factors.

On one hand, some scholars have pointed out that deep learning is influenced by the characteristics of academic personnel, characteristics of college students, academic activities, teaching processes, learning processes, and classroom atmosphere (Sadeghi et.al. 2012). While other scholars also conducted in-depth interviews with 12 undergraduates from different disciplines to study the impact of different learning environments on deep learning. It was found that time commitment, effort, curriculum objectives, and adaptability of learning strategies were key factors affecting deep learning (Postareff et al. 2015). Domestic studies have pointed out that problem-based learning, task-driven learning and procedural evaluation can promote students' deep learning. However, some studies have analyzed 459 documents collected from the 2005-2015 database of foreign countries in the Web of Science database and found that the overall attention to deep learning process is insufficient (Zhang Siqi et al. 2016). Foreign scholars have conducted more extensive research on internal factors that affect deep learning, and domestic scholars have done less research on internal factors of deep learning. However, the research on the internal influence factors of deep learning has mainly remained in theoretical discussion, and relatively few researches concern with learning practice.
On the other hand, some foreign scholars have realized that information technology and the emerging online learning platform have a secondary role in deep learning. Taking online platforms, podcasts, and private broadcast classes as the starting point, research is being conducted on the external factors that affect deep learning. There are studies to select students from different disciplines, and to analyze the positive and negative factors of the deep learning method in the "student-centered" learning environment. It is found that students in the humanities field tend to adopt the deep learning method (Baeten et.al. 2010). Some scholars have found that students can use online learning platforms such as Moodle and Blackboard to conduct online learning activities such as reflection, analysis, and critical thinking, which is helpful to improve deep learning ability (Razzak 2014). Some scholars have also investigated hundreds of first-year undergraduate chemistry majors and analyzed the impact of podcasting on students' test scores. It was found that deep learning methods could not only promote positive understanding of meaning, enhance learning outcomes and academic performance, could also help retain knowledge more consistently (Bartle \& Longnecker 2015).

Some scholars have also found that although the information environment can indeed increase the breadth of information, it does not play a significant role in promoting the depth of cognition and cannot guarantee the efficiency of deep learning (Duan Jinju, Yu Shengquan 2014). Regrettably, although studies have pointed out that information age can increase the breadth of information, but it neither exert enough power on cognition, nor provide a persuasive explanation for its reasons. In summary, current research on the external factors influencing deep learning is mostly based on the learning environment. Although foreign scholars have adopted empirical research methods, the depth and breadth of research needs to be strengthened.

\section{APPLICATION OF DEEP LEARNING IN INTERDISCIPLINARY FIELDS}

\section{A. Research on Deep Learning in the Field of Learning Science}

In recent years, deep learning has been paid attention to in various fields and a large number of related studies have been conducted, with the field of learning science no exception. Learning science is a new science for research and learning. It is an interdisciplinary field covering many multidisciplinary areas such as cognitive science, educational psychology, computer science, sociology, anthropology, and neuroscience (Sawyer 2006). In light of the interdisciplinary nature of learning science, deep learning in the field of learning science reflects a clear interdisciplinary character.

The study of deep learning in the field of learning science is mainly reflected in the theoretical level. Some researchers propose that the main theoretical foundations of deep learning in the field of learning science include: constructivist learning theory, situational cognitive theory, distributed cognitive theory, connectedness learning theory, and metacognitive theory. Among them, constructivism provides a scientific basis for knowledge construction and conceptual interaction. 
Situational cognitive theory provides a powerful explanation for the learning environment and personal learning space design. Distributed cognitive theory and connectivism lay the foundation for deep interaction and group meaning construction. Cognitive theory provides the basis for reflection and self-monitoring (Duan Jinju, Yu Shengquan 2013). They further pointed out that compared with other learning theories, constructivism and connectivism have more direct and farreaching effects on deep learning (ibid.). Unfortunately, because the study focuses on the theoretical level, it is not clear why constructivism and connectivism have a greater impact on deep learning, nor does it explain how the two theories in practice affect deep learning.

With regards to the relationship between deep learning and learning science, some scholars have suggested that deep learning is the key to learning science, and high-level thinking is the core of deep learning (Duan Jinju, Yu Shengquan 2013). Unfortunately, the study did not explore the deep relationships between learning science, deep learning, and higher-order thinking. The author believes that critical thinking is a means to achieve deep learning, the cultivation of critical thinking ability and other higher-order thinking ability is the goal of deep learning, while deep learning research is the focus of learning science research.

In summary, the study of deep learning in the field of learning science focuses mainly on the theoretical level. There is relatively little research on the practical level and the relevant empirical research is scarce. In general, the depth of research on deep learning mode, deep learning environment, and deep learning effectiveness is not enough, and the study on the relationship between deep learning mode, environment, and effectiveness is still relatively weak.

\section{B. Research on Deep Learning in Foreign Language Learning}

Some studies have suggested that surface learning refers to mechanical and passive learning, to memorizing knowledge and details that are not strongly related to each other. However, deep learning focuses on how to promote learners' active learning and emphasizes the connection between knowledge and details (Liu Huiying 2016). Studies have found that teachers play an important role in motivating students to adopt deep learning methods. The more students like the teaching methods of their teachers, the more they will use deep learning (Baeten \& Kyndt 2010). Some scholars have applied deep learning to the field of foreign language learning, proposed the concept of deep foreign language learning, and explained the subject of foreign language learning. We advocate learners' autonomy and intersubjectivity, and give play to their scaffolding role (Tochon 2014). Domestic research also explores the subject of foreign language learning and suggests that "the deep learning approach gives students a dominant role in the construction of the curriculum. The role of the teacher is transformed into a learning facilitator, providing students with a wide range of learning resources and timely and comprehensive feedback" (Liu Huiying 2016:143). In general, the concept of deep foreign language learning emphasizes the role of learners, and reinforces the "scaffolding" role teachers.
Both "teacher-centered" or "student-centered" philosophy emphasizes the role of teachers and students as single subjects, but ignore the interaction between them. At the level of learning and practice, with the continuous advancement of learning cognitive research and changes in learning methods in the digital era, teachers and students are required to give full play to the intersubjective nature, and in-depth learning is implemented dynamically and flexibly according to actual conditions. On one hand, teachers need to deeply understand the characteristics of learners, such as personality, cognitive style, learning methods, purpose of learning, personalized learning experience, and etc., and carry out personalized teaching; on the other hand, learners should be responsible for their own learning and really play a role in learning. The questions such as what to learn, when to learn, how to learn, where to study, how much to learn, and how fast to learn are mainly determined by the learners themselves. Teachers provide timely help when needed. In short, in-depth learning highlights the enthusiasm, initiative and learning drive of the subject, advocates interaction between subjects, cooperates in the completion of new knowledge construction, highlights the intersubjectivity, and advocates interaction and mutual promotion between subjects.

\section{ENLIGHTENMENT OF DEEP LEARNING FOR FOREIGN LANGUAGE LEARNING IN CHINA}

This study refers to deep learning, combines the current research status of foreign language learning and existing problems of academic separation, and conducts research from several aspects including the theoretical level, the subject of learning, and the way of learning. Learning theory plays a guiding role in learning and practice. Learning and practice are both the starting point and the end point of foreign language learning. The subject of learning is the intermediate force linking learning theories and learning practice. It plays a decisive role in the effectiveness of foreign language learning.

\section{A. Theoretical Enlightenment}

Some studies pointed out that great achievements have been made in China's foreign language education, while meanwhile it is facing many problems such as lack of macroscopic thinking and overall planning. It is necessary to carry out an all-round, three-dimensional and systematic research from the perspectives of curriculum, teaching, and learning (Wang Wenbin, Li Min 2017). In view of the outstanding academic separation in the field of foreign language learning, domestic scholars have made many explorations and made important breakthroughs at the theoretical level. Wen Qiufang (2017) proposed the output driven theory with Chinese characteristics. The starting point and the goal of these theories are all related to the problem of the separation of learning and application in China. Deep learning emphasizes the application of linguistic knowledge and the solution to practical problems. It is not difficult to see that deep learning theory has similarities with the "output-driven approach". In other word, deep learning at the theoretical level has guiding significance and enlightenment to our foreign language learning.

Surface foreign language learning focuses on the passive memory of language knowledge, while deep foreign language 
learning promotes active learning, emphasizes the application of language knowledge and skills, understanding and meaning construction. However, as far as the foreign language learning itself is concerned, vocabulary mastery, understanding of language knowledge, memory, and other surface learning are the prerequisites and foundations for deep language learning such as language use and innovation. However, language use is not only the goal of foreign language learning but also the understanding of language knowledge. With effective means of application. Therefore, to achieve foreign language learning goals, it is necessary to integrate surface and deep learning, strengthen "learning to use", and ultimately realize the interaction and mutual promotion between deep learning and surface learning.

At the theoretical cognitive level, we should avoid misunderstandings of knowledge and avoid separating the deep learning from surface learning. For foreign language learning, while deep learning and surface learning have different focuses, in essence, they are inclusive and mutually reinforcing. Combining the characteristics of foreign language learning in the digital age, the level of cognitive development of students, and the demanding orientation of the social and cultural environment for foreign language proficiency, the interdisciplinary perspective of the integration of deep learning and surface learning will be the focus of future studies.

\section{B. Application}

Just as there is no perfect teaching method in the world, there is no universal learning method. In spite of this, it is sure to find a way that is more suitable for students, courses and learning objectives. Some studies have found that deep learning and surface learning have their own advantages and disadvantages and present complementary relationships. There are studies comparing and analyzing the deep learning and surface learning models proposed by Biggs's. They find that the two have their own advantages and disadvantages, and they all have deficiencies. Afterwards, the two conducted a comparative analysis of the conversion learning method and the deep learning method and found that both are also of their own merits (Howie \& Bagnall 2015). Therefore, some researchers suggest that the ideal approach is to effectively integrate deep learning with surface learning, and to achieve an efficient transition from surface learning to deep learning (Duan Jinju, Yu Shengquan 2014). Unfortunately, because of its focus on macro-theory, the study did not explain how to achieve the transition from surface learning to deep learning, as well as specific strategies for the integration of the two.

There are studies that try to explore practical ways to achieve deep learning. Since any knowledge is not isolated, learners need to connect new knowledge with existing knowledge and experience, and build new knowledge based on the original knowledge structure to achieve deep learning. There are also studies that combine foreign language learning to explore the relationship between deep foreign language learning and surface foreign language learning. In general, they are critical of surface learning and have a positive attitude toward deep learning. For example, some studies have suggested that surface learning, such as repetitive exercise and mechanical memory, is a common problem in foreign language education today, which seriously affects the development of critical and innovative thinking among language learners (Liu Huiying 2016).

In terms of foreign language learning, how to correctly view deep learning and surface learning? How to deal with the relationship between the two? These are questions that deserve deep thought. In recent years, the foreign language learning community has paid attention to language exercises and memory, while ignoring high-level thinking such as creative thinking, language use, and problem solving, resulting in weak output capability and severe separation of learning. In spite of this, it should not be an overall negative attitude to surface learning, and it should not be simply to separate surface learning from deep learning. The author believes that since surface learning is the basis of in-depth foreign language learning, achieving the complementary advantages of deep learning and surface learning, as well as the integration and mutual promotion of the two, is the focus of current in-depth foreign language learning research.

At the level of learning and practice, according to the actual situation of students, we can adopt suitable deep learning methods dynamically and flexibly. The effect of deep foreign language learning is influenced by many factors. For example, who is the learner? What language knowledge has been mastered? What language skills are already in place? What kind of learning style? What kind of learning strategies have been mastered? What are the goals for learning in the medium and long term? All of these factors will affect whether students adopt, when and how to use deep learning strategies. Specifically, even if learners learn to understand, internalize, and apply what they have learned, they eventually learn to do things in a foreign language. However, there is still lack of empirical and systematic research on the above issues and indepth foreign language learning methods in the information age. Combining artificial intelligence and big data to conduct systematic research on deep foreign language learning will be another focus of future research.

\section{CONCLUSION}

To improve the effectiveness of in-depth foreign language learning, it is necessary to make use of the complementary and interactive roles of surface and deep learning. The intersubjectivity of both teachers and students should be brought into play, and the interaction between teaching and learning should be promoted. Through combing the literature, the research found that that researchers mostly discuss the application of deep learning in foreign language teaching from the theoretical and macro perspectives, and have relatively few practical explorations of the specific implementation strategies, methods, and implementation effects of deep learning. In addition, combining the latest developments in big data, artificial intelligence, etc., relevant empirical and action research on different stages of learning, for different learners, in different professional courses, and in different learning fields will be a new direction for future studies. 


\section{ACKNOWLEDGMENT}

Learning science was first proposed by Sawyer (2006) in the Cambridge Handbook of Learning Science. It was defined as a new science for research and learning. It belongs to an interdisciplinary category covering cognitive science, educational psychology, computer science, and sociology, anthropology, neuroscience, and many other fields.

\section{Funded Project:}

The paper was funded by the 2017 Philosophy and Social Sciences Project of Zhejiang Province (Project number: 17NDJC292YB), 2018 Philosophy and Social Sciences Project of Zhejiang Province (Project number: 18NDJC284YB), and the Postgraduate Research and Innovation Fund of Sichuan University (Project number: 2012017yjsy134).

About the author:

Wang Honglin (1978- ), Associate professor of Zhejiang Wanli University, Phd Candidate of Sichuan University,

Research areas: Applied linguistics, translation and interpreting studies

Address: Wangjiang campus of Sichuan University, No.24 South Section 1, Yihuan Road, Chengdu, China, 610065

Email: fycarol61@163.com

\section{REFERENCES}

[1] Baeten, M., Kyndt, E., Struyven, K., Doch, F. 2010. Using student centred learning environments to stimulate deep approaches to Teaming Factors encouraging or discouraging their effectiveness[J]. Educational Research Review, (5): 243-260.

[2] Harvey, A., \& Kamvounias, P. 2008. Bridging the implementation gap: a teacher-as-learner approach to teaching and learning policy $[\mathrm{J}]$. Higher Education Research and Development, 27(1), 31-41. doi:10.1080/07294360701658716.

[3] Hinton, G., Deng, L., Dahl, G. E., Mohamed, A., Jaitly, N., Senior, A., Vanhoucke, V., Nguyen, P., Sainath, T., and Kingsbury, B. 2012. Deep neural networks for acousticmodeling in speech recognition[J]. IEEE Signal Processing Magazine, 29(6), 82-97.
[4] Howie, P., Bagnall, R. 2015. A critical comparison of transformation and deep approach theories of learning [DB/OL]. [2017-2-19]. http://dx.doi.org/10.1080/02601370.2014.1000409

[5] Marton F, Saljo R. 1976. On Qualitative Differences in Learning: IOutcome and Process [J]. British Journal of Educational Psychology, 46(1): 4-11.

[6] Postareff, L., Parpala, A., Lindblom-Ylänne, S. 2015. Factors contributing to changes in a deep approach to learning in different learning environments. In: Learning Environ Res 18: 315-333. DOI 10.1007/s10984-015-9186-1

[7] Razzak, N. A. 2014. Strategies for effective faculty involvement in online activities aimed at promoting critical thinking and deep learning[J]. Education and Information Technologies, 19(4). DOI: 10.1007/s10639-014-9359zhttps://www.researchgate.net/publication/266202067

[8] Sadeghi A, et.al. 2012. The Factors Affecting University Student Deep Learning(USDL) in the University of Guilan, IRAN(comparative study among Humanities, Agricultural and physical Education Faculties)[J]. Procedia-Social and Behavioral Sciences, (31): 810-815.

[9] Sawyer, K. 2006. Introduction: The new science of learning[A]. Sawyer, R. K. (Eds.).Cambridge handbook of the learning sciences[C]. New York: Cambridge UniversityPress: 1-18.

[10] Tochon, F.V. 2014. Help Them Learn a Language Deeply[M]. Blue Mounds: Deep University Press.

[11] Duan Jinju, Yu Shengquan. 2013. Overview of Study on Deep Learning with E-learning Based on Learning Science[J]. Journal of Distance Education, (4): 43-51. (In Chinese)

[12] Jiao Jianli, Jia Yimin. 2011. New Progress of Learning Sciences: A Research Agenda[J]. Open Education Research. (In Chinese)

[13] Liu Huiying. 2016. On the Deep Approach to Foreign Language Learning: A Curriculum Perspective[J]. Journal of Guangdong University of Foreign Studies, (2): 138-144.(In Chinese)

[14] Liu Jianwei, Liu Yuan, Luo Xiongling. 2014. Research Progress in Deep Learning[J]. Application Research of Computers, (7): 1921-1930. (In Chinese)

[15] Wang Wenbin, Li Min. 2017. Theoretical Framework of Foreign Language Education Research: Construction and Expounding[J]. Foreign Language Education, (1): 1-6. (In Chinese)

[16] Wen Qiufang. 2017. Chinese Features Displayed in the Productionoriented Approach[J]. Modern Foreign Languages, (3): 348-358. (In Chinese)

[17] Zhang Siqi, Zhang Wenlan, Libao. 2016. Focus and Trend of Deep Learning Research in Foreign Countries in Recent Ten Years: Based on Citation Analysis and the Knowledge Map Analysis of Co-word Matrix[J]. Journal of Distance Education, (2): 64-72. 